Draft VERSion MAY 21, 2018

Preprint typeset using IATEX style emulateapj v. 5/2/11

\title{
REVISITING ACCELERATION OF CHARGED GRAINS IN MHD TURBULENCE
}

\author{
Thiem HoAng $^{1}$, A. LAzarian ${ }^{1}$, \& R. Schlickeiser ${ }^{2}$ \\ ${ }^{1}$ Department of Astronomy, University of Wisconsin, Madison, WI 53706, USA \\ ${ }^{2}$ Institut für Theoretische Physik, Lehrstuhl IV: Weltraum- und Astrophysik, Ruhr-Universität Bochum, 44780 Bochum, Germany \\ Draft version May 21, 2018
}

\begin{abstract}
We study the acceleration of charged grains by magnetohydrodynamics (MHD) turbulence in the interstellar medium (ISM). We begin with revisiting gyroresonance acceleration by taking into account the fluctuations of grain guiding center along a uniform magnetic field (i.e. nonlinear theory-NLT). We calculate grain velocities due to gyroresonance by fast MHD modes using the NLT for different phases of the ISM, and compare with results obtained using quasi-linear theory (QLT). We find for the parameters applicable to the typical ISM phases that the fluctuations of grain guiding center reduce grain velocities by less than $15 \%$, but they can be important for more special circumstances. We confirm that large grains can be accelerated to super-Alfvénic velocities through gyroresonance. For such super-Alfvénic grains, we investigate the effect of further acceleration via transit time damping (TTD) by fast modes. We find that due to the broadening of resonance condition in the NLT, the TTD acceleration is not only important for the cosines of grain pitch angle relative to the magnetic field $\mu>V_{\mathrm{A}} / v$, but also for $\mu<V_{\mathrm{A}} / v$ where $v$ is the grain velocity and $V_{\mathrm{A}}$ is the Alfvén speed. We show that the TTD acceleration is dominant over the gyroresonance for large grains, and can increase substantially grain velocities induced by gyroresonance acceleration. We quantify another stochastic acceleration mechanism arising from low frequency Alfvén waves. We discuss the range of applicability of the mechanisms and their implications.

Subject headings: dust, extinction - ISM: kinematics and dynamics, acceleration - ISM
\end{abstract}

\section{INTRODUCTION}

Dust grains play crucial roles in many aspects of the interstellar medium (ISM). For example, alignment of dust grains with respect to magnetic field provides insight into star formation through far-infrared and submm polarized emission (see Lazarian 2007 for a review). Very small spinning dust grains radiate microwave emission that contaminate to cosmic microwave background (CMB) radiation (Draine \& Lazarian 1998; Hoang, Draine \& Lazarian 2010; Hoang, Lazarian \& Draine 2011). Optical extinction and polarization properties of dust depend mainly on its size distribution. Grain-grain collisions, which depend on grain relative motions, govern grain coagulation and destruction, that result in the grain size distribution (see Hirashita \& Yan 2009). Grain-grain collisions are considered the first stage of planetesimal formation in circumstellar disks (see e.g., Dullemond \& Dominik 2005).

Traditionally it is believed that the motion of dust grains in the ISM arises from radiative force, ambipolar diffusion and hydrodrag (see Draine 2011). The resulting motion from these processes is sub-Alfvénic (i.e., $v \ll V_{\mathrm{A}}=B / \sqrt{4 \pi \rho}$ where $B$ is the magnetic field strength and $\rho$ is the gas mass density), except in special environment conditions (see Purcell 1969; Roberge et al. 1993)

Astrophysical environments are practically all magnetized and turbulent, and turbulence is expected to be an important factor in accelerating dust grains. The evidence for turbulence from electron density fluctuations testifies for the existence of the so-called Big Power Law in the Sky (Armstrong et al. 1995; Chepurnov \& Lazarian 2010), while the fluctuations of velocity (see Lazar- ian 2009 and references therein) provide convincing evidences of the dynamic nature of the observed inhomogeneities. Whether an environment is thermally dominated or magnetized dominated depends on the plasma $\beta$ parameter, which is defined as the ratio of gas pressure to magnetic pressure

$\beta=\frac{8 \pi n_{\mathrm{H}} k_{\mathrm{B}} T_{\mathrm{gas}}}{B^{2}}=0.1\left(\frac{n_{\mathrm{H}}}{30 \mathrm{~cm}^{-3}}\right)\left(\frac{T_{\mathrm{gas}}}{100 \mathrm{~K}}\right)\left(\frac{10 \mu G}{B}\right)^{2}$

where $n_{\mathrm{H}}$ is the gas density and $T_{\text {gas }}$ is the gas temperature.

Recent decade has been marked by substantial progress in understanding of MHD turbulence. This included generalizing incompressible Alfvénic turbulence ${ }^{1}$ by Goldreich \& Sridhar (1995) to realistically compressible media and successful testing of the compressible theory (Lithwick \& Goldreich 2001; Cho \& Lazarian 2002, 2003; Kowal \& Lazarian 2010). In what follows in describing compressible MHD turbulence we shall be guided by the mode decomposition of MHD turbulence into Alfvén, slow and, fast modes presented in Cho \& Lazarian (2002, 2003).

Studies of grain acceleration for magnetized turbulent environments were initiated by Lazarian \& Yan (2002) who dealt with the acceleration by incompressible Alfvénic turbulence. Comprehensive studies of the acceleration in realistically compressible environments were performed in Yan \& Lazarian (2003, hereafter YL03) and

${ }^{1}$ While there are still ongoing debates about the detailed structure and dynamics of incompressible MHD turbulence, we believe that Goldreich \& Sridhar (1995) model provides an adequate starting point. In fact, recent studies in Beresnyak \& Lazarian (2010) and Beresnyak (2011) provided additional supports for the model. 
Yan, Lazarian \& Draine (2004, hereafter YLD04). Those studies identified gyroresonant interactions of grains with fast MHD modes as a new powerful mechanism of grain acceleration. Recently, Yan (2009) considered betatron acceleration and came to the conclusion that for most environments the betatron acceleration is subdominant to the gyroresonance acceleration for sub-Alfvénic grains. The application of grain velocities predicted by gyroresonance for modeling dust extinction curve provided good correspondences between observations and theoretical predictions (Hirashita \& Yan 2009) indicating that the turbulence is indeed the main driving force behind grain acceleration.

The gyroresonance acceleration due to compressible MHD turbulence (YL03;YLD04) was studied using quasi-linear theory (QLT, Jokipii 1966; Schlickeiser \& Miller 1998). The underlying assumption of the QLT is that the guiding center is assumed to move in a regular trajectory along a uniform magnetic field $\mathbf{B}_{0}$. The condition for a grain with velocity $v$ to resonantly interact with fast MHD modes at the scale $k_{\|}$is given by $\omega-k_{\|} v \mu-\omega=n \Omega$, for $n=0, \pm 1, \pm 2, \ldots$ where $\mu$ is the cosine of the grain pitch angle between $\mathbf{v}$ and $\mathbf{B}_{0}, \omega$ is the wave frequency, and $\Omega$ is the Larmor frequency of the charged grain around $\mathbf{B}_{0}$. The gyroresonance acceleration with $n \neq 0$ is dominant by eddies with size equal to gyro radius $l \sim r_{g}$.

Transit-time damping (TTD) or transit-time acceleration, arises from resonant interactions of particles with the compressive component of magnetic fluctuations (i.e., the component parallel to the mean magnetic field $\mathbf{B}_{0}$ ). When the grain moves together with the wave along $\mathbf{B}_{0}$, it is subject to magnetic mirror forces $-\left(m v_{\perp}^{2} / 2 B\right) \nabla_{\|} \mathbf{B}$, where $m$ is the grain mass, $v_{\perp}$ is the grain velocity component perpendicular to $\mathbf{B}_{0}$, and $\mathbf{B}$ is the total magnetic field. In the plasma reference, the back and forth collisions of the grain with the moving magnetic mirrors increase grain energy because the head-on collisions are more frequent than trailing collisions due to the larger relative velocity between grain and wave (see Fisk 1976; Schlickeiser \& Miller 1998). The TTD acceleration with resonance condition $k_{\|} v \mu=\omega$, was disregarded in previous studies on grain acceleration because grains are expected to move slowly along the uniform magnetic field, for which they can not catch up with the propagation of magnetic mirrors along this direction. Although the gyroresonance is found to be able to accelerate large grains to super-Alfvénic velocities (see YL03; YLD04), which is sufficient to trigger TTD, the resulting grain motion mostly perpendicular to the uniform field $\mathbf{B}_{0}$ (i.e. $v \mu=0)$ in the QLT regime makes TTD incapable.

Due to magnetic fluctuations in the ISM, the local magnetic field $\mathbf{B}$ can be decomposed into a uniform field plus a turbulent component, i.e., $\mathbf{B}=\mathbf{B}_{0}+\delta \mathbf{B}$. Thus, any perturbation $\delta \mathbf{B}$ will induce the fluctuations of grain guiding center from a regular trajectory along the uniform magnetic field (see e.g., Shalchi 2005). Non-linear theory (hereafter NLT) for gyroresonance that takes into account such fluctuations of guiding center was formulated in Yan \& Lazarian (2008, hereafter YL08) to describe the propagation of energetic particles, and later it was applied in Yan et al. (2008, hereafter YLP08) to study acceleration of energetic particles in solar flares.

The important modification present in the NLT is the broadening of resonance function from a Delta function $\delta\left(\omega-k_{\|} v_{\|}-n \Omega\right)$ to a Gaussian function $R_{n}\left(\omega-k_{\|} v_{\|}-n \Omega\right)$ (see YL08). Such a broadening of the resonance condition allows grains moving with $v \sim V_{\mathrm{A}}$ perpendicular to $\mathbf{B}_{0}$ to have TTD with compressive waves propagating along $\mathbf{B}_{0}$. We are going to clarify the effects that TTD induces on grain acceleration in the present paper.

In what follows, we revisit the basics of resonance acceleration for charged grains by taking into account additional physical processes that were not considered within original treatments. In discussing the gyroresonance acceleration, we are going to take into account the fluctuations of grain guiding center. In particular, we are going to investigate the efficiency of TTD on grain acceleration in MHD turbulence.

The structure of the paper is as follows. In $\S 2$, we present briefly the problem of grain charging, important dynamical timescales, and identify the range of grain size in which grain charge fluctuations are important. We revisit gyroresonance acceleration, and introduce TTD acceleration in $\S 3$. Grain velocities induced by gyroresonance acceleration and TTD are presented in $\S 4$. $\$ 5$ is devoted for stochastic acceleration by low frequency Alfvén waves. Discussion and summary are presented in $\S 6$ and 7 , respectively.

\section{GRAIN CHARGING AND DYNAMICS}

\subsection{Grain Charging}

Charging processes for a dust grain in the ISM consist of its sticking collisions with charged particles in plasma (Draine \& Sutin 1985) and photo-emission induced by $h \nu \geq 13.6 \mathrm{eV}$ photons (Weingartner \& Draine 2001). In the former case, the grain acquires charge by capturing electrons and ions from the plasma, while in the latter case the grain looses charge by emitting photoelectrons. After a sufficient time, these processes result in a statistical equilibrium of ionization, and the grain has a mean charge, denoted by $\langle Z\rangle$, which is equal to the charge averaged over time. Due to the discrete nature of charging events, the grain charge fluctuates around $\langle Z\rangle$. The probability of finding the grain with charge $Z e$ is described by charge distribution function $f_{Z}$. Here we find the charge distribution $f_{Z}$ using statistical ionization equilibrium as in Draine \& Sutin (1985) and Weingartner \& Draine (2001). Hoang \& Lazarian (2011) found that the statistical ionization equilibrium is not applicable for tiny grains with size $a<10 \AA$ for which the charging is infrequent, but it is adequate for grains considered in the present paper.

Figure 2 shows the variation of the grain mean charge $|\langle Z\rangle|$ for graphite and silicate grains in the cold neutral medium (CNM), warm neutral medium (WNM), warm ionized medium (WIM). In the WIM, $\langle Z\rangle$ varies rapidly with the grain size, and change its sign at $a \sim 10^{-6}$ and $\sim 10^{-5} \mathrm{~cm}$, marked by filled circles.

Let us define a characteristic relaxation time of the charge fluctuations, $\tau_{Z}$, which is equal to the time required for the grain charge to relax from $Z$ to the equilibrium state (Draine \& Lazarian 1998b):

$$
\tau_{Z}=\frac{\left\langle(Z-\langle Z\rangle)^{2}\right\rangle}{\sum_{Z} f_{Z} J_{t o t}(Z)} \equiv \frac{\sigma_{Z}^{2}}{\sum_{Z} f_{Z} J_{\text {tot }}(Z)},
$$

where $J_{\text {tot }}(Z)$ is the total charging rate due to collisional 


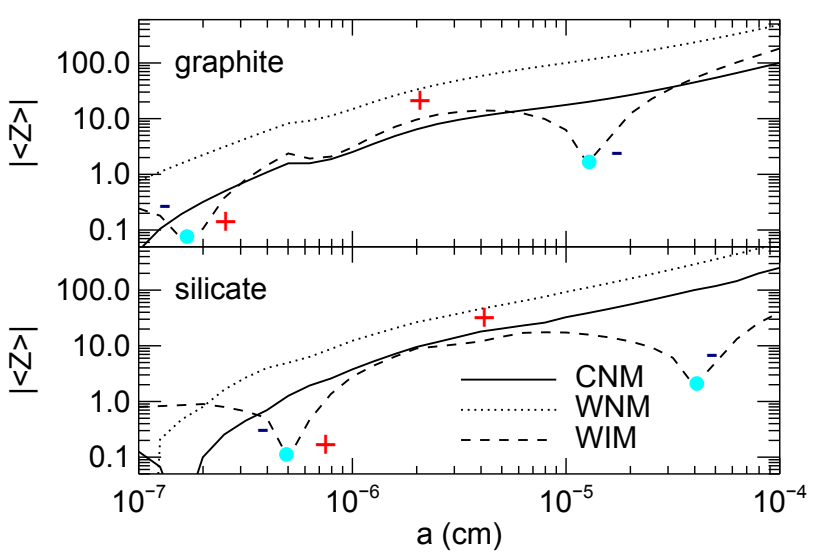

FIG. 1.- Mean grain charge $|\langle Z\rangle|$ as functions of grain size $a$ for graphite and silicate grains in different ISM phases. For the WIM, $|\langle Z\rangle|$ changes rapidly with $a$, and filled circles mark the change in grain charge between being positively charged $(+)$ and negatively charged (-).

charging and photoemission (see Draine \& Sutin 1987; Weingartner \& Draine 2001). Here we averaged over all possible charge states $Z$ to find $\tau_{Z}$.

In an ambient magnetic field $\mathbf{B}$, the grain with mean charge $\langle Z\rangle e$ gyrates about $\mathbf{B}$ on a timescale equal to the Larmor period:

$\tau_{L}=\frac{2 \pi m c}{|\langle Z\rangle| e B}=1.56 \times 10^{2}\left(\frac{a}{10^{-6} \mathrm{~cm}}\right)\left(\frac{\mu G}{|\langle Z\rangle| B}\right) \mathrm{yr}$,

where $m=4 / 3 \pi a^{3} \rho_{d}$ with $\rho_{d}$ being the dust mass density is the grain mass. We adopt $\rho_{d}=2.2$ and $3.0 \mathrm{~g} \mathrm{~cm}^{-3}$ for graphite and silicate grains, respectively. The Larmor frequency reads $\Omega=(\langle Z\rangle e B) / m c$.

We calculate the relaxation time of charge fluctuations $\tau_{Z}$ for both graphite and silicate grains in various phases of the ISM with physical parameters listed in Table 1. Figure 2 compares $\tau_{Z}$ with the gas drag time $\tau_{\text {drag }}$ (see Eq. 5) and the Larmor period $\tau_{L}$. It can be seen that $\tau_{Z} \ll \tau_{L}<\tau_{\text {drag }}$ for grains larger than $\sim 2 \times 10^{-7} \mathrm{~cm}$. For grains smaller than $\sim 2 \times 10^{-7} \mathrm{~cm}, \tau_{Z} \geq \tau_{L}$, so that the assumption for grains to have a constant charge is no longer valid. As a result, the fluctuations of grain charge should be accounted for in the treatment of resonance acceleration for such very small grains. This issue will be addressed in our future paper, in which we employ Monte Carlo method to simulate grain charge fluctuations (see e.g., Hoang \& Lazarian 2011). In the present paper, for the sake of simplicity, we adopt $\langle Z\rangle e$ for grain charge within the entire range of the grain size distribution.

\subsection{Grain Translational Damping}

Interactions of dust grains with the ambient gas present the primary mechanism of dissipating translational motions of grains. The damping rate of translational motion arising from the interaction with neutral gas is essentially the inverse time for collisions with the mass of the gas equal that of a grain (Purcell 1969),

$$
\begin{aligned}
\tau_{d n}^{-1} & =\sqrt{\frac{8}{\pi}} \frac{n_{n}}{a \rho_{d}}\left(m_{n} k_{\mathrm{B}} T_{n}\right)^{1 / 2}, \\
& =2.4 \times 10^{-12}\left(\frac{10^{-6} \mathrm{~cm}}{a}\right)\left(\frac{n_{n}}{30 \mathrm{~cm}^{-3}}\right)\left(\frac{T_{n}}{100 \mathrm{~K}}\right)^{1 / 2} \mathrm{~s}^{-1},
\end{aligned}
$$

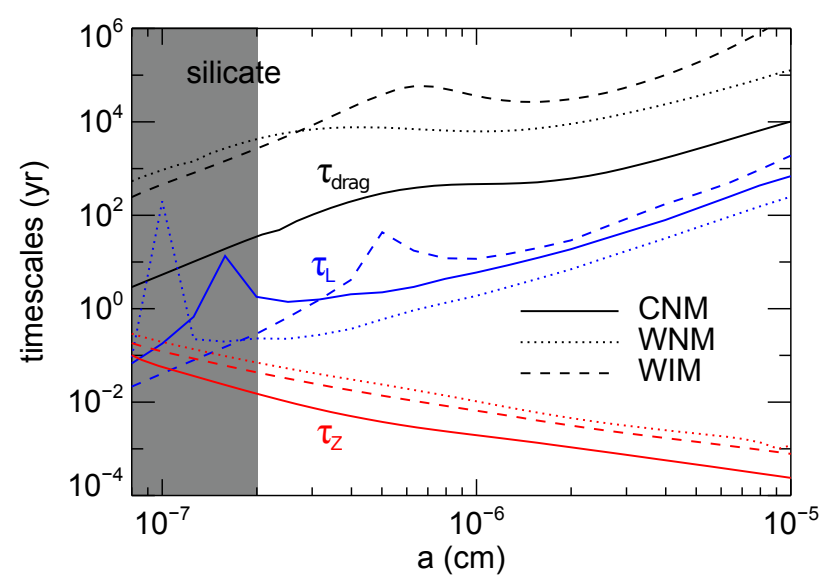

FIG. 2.- Timescales for gas drag $\tau_{\text {drag }}$, Larmor period $\tau_{L}$, and charge fluctuations $\tau_{Z}$ as functions of the grain size $a$ for subsonic silicate grains in the various phases of the ISM. Shaded area marks the range of grain size in which the charge fluctuations are important, i.e., $\tau_{Z} \geq \tau_{L}$. The peaks in $\tau_{L}$ correspond to the change in sign of grain charge.

where $m_{n}, n_{n}$, and $T_{n}$ are the mass, volume density, and temperature of neutrals, and $a$ is the grain radius.

When the ionization degree is sufficiently high, the interaction of charged grains with the plasma becomes important. The ion-grain cross section due to long-range Coulomb forces is larger than the atom-grain cross section. As a result, the rate of translational motion damping gets modified. For subsonic motions the effective damping time due to gas drag is renormalized:

$$
\tau_{\text {drag }}=\alpha^{-1} \tau_{d n}
$$

with the following renormalizing factor (Draine \& Salpeter 1979)

$$
\begin{gathered}
\alpha=1+\frac{n_{\mathrm{H}}}{2 n_{n}} \sum_{i} x_{i}\left(\frac{m_{i}}{m_{n}}\right)^{1 / 2} \sum_{Z} f_{Z}\left(\frac{Z e^{2}}{a k_{\mathrm{B}} T_{i}}\right)^{2} \\
\times \ln \left[\frac{3}{2|Z| \sqrt{\pi x n_{\mathrm{H}}}}\left(\frac{k_{\mathrm{B}} T_{i}}{e^{2}}\right)^{3 / 2}\right] .
\end{gathered}
$$

Here $x_{i}$ is the abundance of ion $i$ (relative to hydrogen) with mass $m_{i}$ and temperature $T_{i}, x=\sum_{i} x_{i}, Z e$ is the grain charge, and $f_{Z}(Z)$ is the grain charge distribution function. When the grain velocity $v_{d}$ relative to gas becomes supersonic, the dust-plasma interaction is diminished, and the damping rate in this case is renormalized due to the gas-dynamic correction (Purcell 1969),

$$
\alpha=\left(1+\frac{9 \pi}{128} \frac{v_{d}^{2}}{C_{\mathrm{s}}^{2}}\right)^{1 / 2}
$$

where $C_{\mathrm{s}}=\sqrt{k_{\mathrm{B}} T_{n} / m_{n}}$ is the sound speed.

If $\tau_{\mathrm{L}}$ is greater than $\tau_{\text {drag }}$, then the effect of magnetic field on dust dynamics is negligible. However, $\tau_{\mathrm{L}} \ll \tau_{\text {drag }}$ in most phases of the ISM.

\section{RESONANCE ACCELERATION}

\subsection{Gyroresonance acceleration: nonlinear theory}

In this section, we revisit the treatment of resonance acceleration by fast modes in compressible MHD turbu(4)ence using nonlinear theory (NLT). 
TABLE 1

IDEALIZED ENVIRONMENTS AND MHD TURBULENCE PARAMETERS

\begin{tabular}{llll}
\hline \multicolumn{1}{c}{ Parameters } & \multicolumn{1}{c}{ CNM } & WNM & WIM \\
\hline$n_{\mathrm{H}}\left(\mathrm{cm}^{-3}\right)$ & 30 & 0.4 & 0.1 \\
$T_{\text {gas }}(\mathrm{K})$ & 100 & 6000 & 8000 \\
$x_{\mathrm{H}}$ & 0.0012 & 0.1 & 0.99 \\
$B(\mu G)$ & 6 & 5.8 & 3.35 \\
$L(\mathrm{pc})$ & 0.64 & 100 & 100 \\
$\delta V=V_{\mathrm{A}}\left(\mathrm{km} \mathrm{s}^{-1}\right)$ & 2 & 20 & 20 \\
$k_{c}\left(\mathrm{~cm}^{-1}\right)$ & $7 \times 10^{-15}$ & $4 \times 10^{-17}$ & $\ldots$ \\
Damping & Neutral-ion & Neutral-ion & Ion viscous \\
& & & and collisionless \\
\hline
\end{tabular}

${ }^{\text {a }}$ Here $n_{\mathrm{H}}$ is the gas density, $T_{\text {gas }}$ is the gas temperature, $x_{\mathrm{H}}$ is the ionization fraction of $\mathrm{H}, B$ is the strength of magnetic field, $L$ is the injection scale of turbulence, $\delta V$ is the rms velocity of turbulence at the injection scale, and $k_{c}$ is the cutoff scale of turbulence due to collisional and collisionless damping.

Consider a grain of mass $m$, charge $Z e$, moving with velocity $v$ in a magnetized turbulent medium with a uniform magnetic field $\mathbf{B}=\mathbf{B}_{0}$. The motion of such charged grain in $\mathbf{B}$ consists of the gyration of the grain about its guiding center and the translation of the guiding center along $\mathbf{B}$. In the QLT limit, the guiding center is assumed to follow a regular trajectory along $\mathbf{B}$ with a constant cosine of pitch angle $\mu=\cos \beta$ with $\beta$ being the angle between $\mathbf{v}$ and $\mathbf{B}$. Gyroresonant interactions between grain and wave occur when the wave frequency in a reference system fixed to the grain guiding center is a multiple of the Larmor frequency:

$$
\omega-k_{\|} v \mu=n \Omega
$$

with $n= \pm 1, \pm 2, \ldots$. This resonance condition is equivalently described by a Delta function $\delta_{n}\left(\omega-k_{\|} v \mu-n \Omega\right)$.

Gyroresonance accelerates grains in the direction perpendicular to the mean magnetic field $\mathbf{B}_{0}$ because electric field induced by plasma perturbations is perpendicular to $\mathbf{B}_{0}$ (see e.g. YLD04). This acceleration mechanism is dominant by eddies smaller than the grain gyroradius, i.e., $l \leq r_{g}$. Indeed, consider gyroresonance by fast MHD modes in low- $\beta$ plasma. From the resonance condition (8) for $n=1$ with $\omega=k_{\|} v_{\mathrm{A}}$, we obtain turbulent scales for gyroresonance $k \geq k_{\text {res }}=r_{g}^{-1}$ or $l \leq r_{g}$, where the fact that $\mu \geq-1$ has been used.

In a turbulent medium, the local magnetic field $\mathbf{B}=$ $\mathbf{B}_{0}+\delta \mathbf{B}$ where $\delta \mathbf{B}$ is the turbulent component of magnetic field, varies both in space and time, so $\mu$ changes, and $v_{\|}$and $v_{\perp}$ change accordingly. The grain guiding center has fluctuations from its regular trajectory along $\mathbf{B}_{0}$. The NLT takes into account such fluctuations of the guiding center.

Assuming that the projection of the fluctuations of grain guiding center onto the mean field $\mathbf{B}_{0}$ can be described by a Gaussian distribution, the resonance condition becomes

$R_{n}\left(\omega-k_{\|} v \mu-n \Omega\right)=\frac{\sqrt{\pi}}{k_{\|} \Delta v_{\|}} \exp \left[-\frac{\left(k_{\|} v \mu-\omega+n \Omega\right)^{2}}{k_{\|}^{2}\left(\Delta v_{\|}\right)^{2}}\right]$, where $n=0$ and \pm 1 , and $\Delta v_{\|}$is the dispersion of velocity (see YL08 and YLP08; Appendix C).

We are interested in the grain acceleration, so the diffusion coefficient arising from gyro-phase averaging $D_{p p}$ is used (see Schlickeiser \& Miller 1998). In compressible MHD turbulence, the fast modes are shown to be dominant in gyroresonance acceleration (YL03). Its corresponding diffusion coefficient is given by (see Appendix C)

$$
\begin{gathered}
D_{p p}(\mu, p)^{\mathrm{G}}=\frac{v \sqrt{\pi} \Omega^{2}\left(1-\mu^{2}\right) m^{2} V_{\mathrm{A}}^{2} M_{\mathrm{A}}^{2}}{4 L R^{2}} \int_{1}^{k_{c} L} x^{-5 / 2} d x \\
\times \int_{0}^{1} \frac{d \eta}{\eta \Delta \mu}\left[J_{0}^{2}(w)+J_{2}^{2}(w)\right] \exp \left[-\frac{\left(\mu-\frac{V_{A}}{\eta v}+n \frac{1}{\eta x R}\right)^{2}}{(\Delta \mu)^{2}}\right],(10)
\end{gathered}
$$

where $n= \pm 1$. In the above equation, $L$ is the injection scale of turbulence, $w=k_{\perp} v_{\perp} / \Omega, x=k / k_{\min }=k L, R=$ $v k_{\min } / \Omega, M_{\mathrm{A}}^{2}=\delta V^{2} / V_{\mathrm{A}}^{2}, \eta=\cos \theta$ with $\theta$ is the angle between the wave vector $\mathbf{k}$ and the mean magnetic field, $k_{c}$ is the cut-off of turbulence cascade due to damping, and $J_{n}$ is second order Bessel function. The dispersion of the cosine of pitch angle $\Delta \mu$ is given by Equation (C5) in Appendix C.

\subsection{Transit-Time Damping (TTD)}

Transit-time damping (TTD) or transit-time acceleration, arises from resonant interactions of particles with the compressive component of magnetic fluctuations, i.e., the component parallel to the mean magnetic field $\mathbf{B}_{0}$ in magnetized turbulent environments. When the grain moves together with the wave along $\mathbf{B}_{0}$, it is subject to magnetic mirror forces $-\left(m v_{\perp}^{2} / 2 B\right) \nabla_{\|} \mathbf{B}$, where $v_{\perp}$ is the grain velocity component perpendicular to $\mathbf{B}_{0}$. In the plasma reference, the back and forth collisions of the grain with the moving magnetic mirrors increase grain energy because the head-on collisions are more frequent than trailing collisions (see e.g., Fisk 1976). The resonance condition for a grain with velocity $\mathbf{v}$ reads

$$
\omega-k_{\|} v_{\|}=0
$$

where $v_{\|}=v \mu$ is the grain velocity component parallel to B, and $\omega$ is the wave frequency (see Fisk 1976; Schlickeiser \& Miller 1998).

For fast MHD modes in low- $\beta$ plasma, the dispersion relation is $\omega=k V_{\mathrm{A}}$ (see Cho et al. 2002), and the required velocity for TTD corresponds to $v_{\|}=v_{\mathrm{A}} / \cos \theta$. Thus, if $v_{\|} \geq V_{\mathrm{A}}$, TTD can be efficient to accelerate grains to large velocities.

In the NLT limit, the diffusion coefficient for TTD is given by (see Appendix C)

$$
\begin{aligned}
D_{p p}(\mu, p)^{\mathrm{TTD}}= & \frac{v \sqrt{\pi} \Omega^{2}\left(1-\mu^{2}\right) m^{2} V_{\mathrm{A}}^{2} M_{\mathrm{A}}^{2}}{2 L R^{2}} \int_{1}^{k_{c} L} x^{-5 / 2} d x \\
& \times \int_{0}^{1} \frac{d \eta}{\eta \Delta \mu} J_{1}^{2}(w) \exp \left[-\frac{\left(\mu-\frac{V_{\mathrm{A}}}{\eta v}\right)^{2}}{(\Delta \mu)^{2}}\right] \cdot(12
\end{aligned}
$$

In Figure 3 we present $D_{p p}^{\mathrm{TTD}}$ as a function of the cosine of the grain pitch angle $\mu$ obtained using the QLT and NLT. Two values of the grain velocity $v=1.5 V_{\mathrm{A}}$ and $3.5 V_{\mathrm{A}}$ are considered. It can be seen that in the former (9) case, $D_{p p}^{\mathrm{TTD}}$ increases with decreasing $\mu$ until $\mu=v / V_{\mathrm{A}}$, 


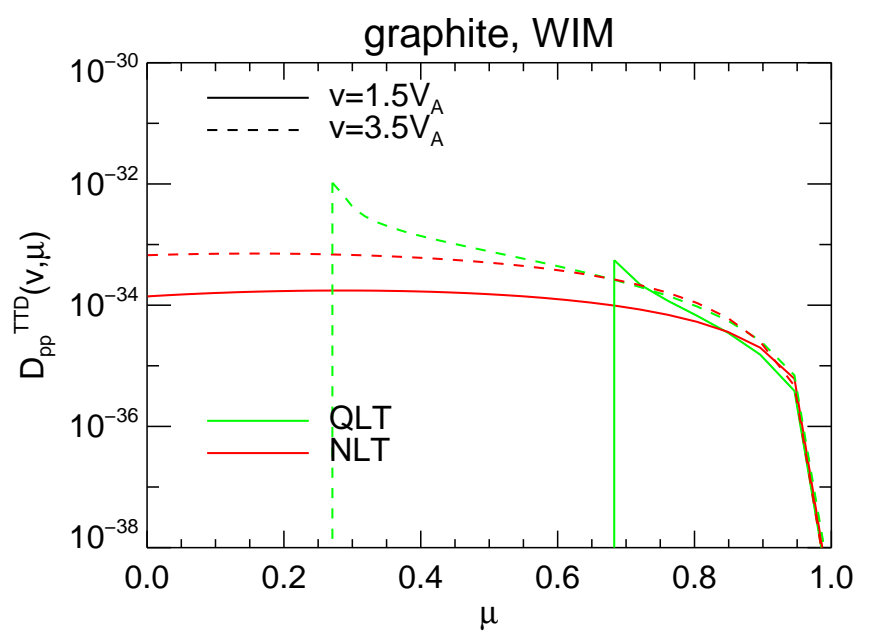

FIG. 3.- Diffusion coefficient $D_{p p}^{\mathrm{TTD}}$ as a function of the cosine of the grain pitch angle $\mu$ for the limit of QLT (green lines) and NLT (red lines). Two velocity values of super-Alfvénic graphite grains of size $a=10^{-5} \mathrm{~cm}$ are considered. $D_{p p}^{\mathrm{TTD}}$ drops sharply for $\mu<V_{\mathrm{A}} / v$ in the QLT, but $D_{p p}^{\mathrm{TTD}}$ is finite as $\mu \rightarrow 0$ in the NLT as a result of broadening of resonance conditions.

and drops sharply to zero for $\mu<v / V_{\mathrm{A}}$ because the resonance condition (11) is not satisfied. In contrast, the broadening of the resonance condition in the latter case allows grains with $\mu<V_{\mathrm{A}} / v$ to have resonant interactions with waves, resulting in finite $D_{p p}^{\mathrm{TTD}}$ even at $\mu=0$. Therefore, TTD is usually neglected in the QLT because the gyroresonance tends to accelerate grains in the perpendicular direction to $\mathbf{B}_{0}$, resulting in $\mu=0$, for which $D_{p p}^{\mathrm{TTD}} \rightarrow 0$. However, TTD can play an important role in driving grain motion when the fluctuations of guiding center are taken into account.

\section{GRAIN VELOCITIES DUE TO RESONANCE ACCELERATION}

\subsection{Grain dynamics}

Consider an ensemble of grains with the same mass $m$, moving in the uniform magnetic field with their different pitch angles $\mu$. From the equation of motion, $m d v / d t=$ $-m v / t_{\mathrm{drag}}+R$, where $R$ is the random force, we can obtain

$$
m \frac{d\left\langle v^{2}\right\rangle}{d t}=-\frac{m\left\langle v^{2}\right\rangle}{t_{\mathrm{drag}}}+A(v),
$$

where $\left\langle v^{2}\right\rangle$ is the grain velocity dispersion averaged over the ensemble of grains, $A(v)$ is the rate of energy gain (see YL03).

When the scattering is less efficient than the acceleration, ${ }^{2}$ the cosine of the grain pitch angle $\mu=\cos \beta$ changes slowly during acceleration, and $A(v)$ is given by

$$
A(v)=\frac{1}{4 p^{2}} \frac{\partial}{\partial p}\left(v p^{2} D_{p p}(p, \mu)\right),
$$

where $D_{p p}(p, \mu)$ is the diffusion coefficient.

\footnotetext{
2 The efficiency of scattering relative to acceleration is described by the ratio $p^{2} D_{\mu \mu} / D_{p p}=\cos \theta^{-2}\left(v \cos \theta / V_{\mathrm{A}}+\mu\right)^{2}$ where $\cos \theta=k_{\|} / k$ with $\theta$ is the angle between $\mathbf{k}$ and $\mathbf{B}$ (see Appendix D). The scattering is negligible for grains with $v<V_{\mathrm{A}}$, but it becomes important for $v \geq V_{\mathrm{A}}$ or when the grain is moving along the magnetic field, i.e., $v_{\|} \gg v_{\perp}$.
}

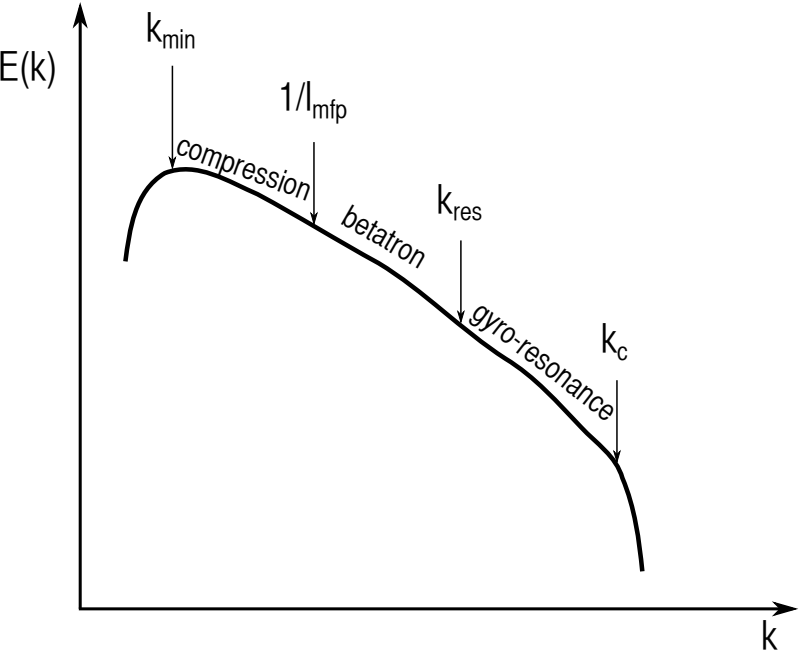

Fig. 4.- A sketch of spectrum of MHD turbulence and acceleration mechanisms for charged grains are shown with its corresponding scale. $k_{\min } \sim L^{-1}$ is the injection scale, $l_{\mathrm{mfp}}$ is the grain mean free path, $k_{\text {res }} \sim r_{g}^{-1}$ is the gyroresonance scale, and $k_{c}$ is the damping cut-off of turbulence. We assume that grains of interest are large enough so that $k_{\text {res }}<k_{c}$ or Larmor period larger than eddy turnover time.

When the scattering is more efficient than the acceleration, the pitch angle can be rapidly redistributed through pitch angle diffusion during the acceleration (i.e., diffusion approximation). The rate of energy gain (Eq. 14) is then determined by the averaged value $D_{p}$ of $D_{p p}(p, \mu)$ over the isotropic distribution of $\mu$ (see Dung \& Schlickeiser 1990ab):

$$
D_{p}(p)=\frac{1}{2} \int_{-1}^{1}\left(D_{p p}(p, \mu)-\frac{D_{\mu p}^{2}(p, \mu)}{D_{\mu \mu}(p, \mu)}\right) d \mu .
$$

We consider in the present paper zero helicity turbulence with $D_{\mu p}=0$. When $D_{p p}$ is known, we solve Equation (13) iteratively to get convergent velocities.

Physical parameters for ISM conditions are shown in Table 1. MHD turbulence is injected at a large outer scale $L$ with velocity dispersion $\delta V$. The value $\delta V$ is chosen such that the turbulence is weak and sub-Alfvénic. Major damping processes are also listed in Table 1. For the CNM and WNM, the dominant damping arises from the neutral-ion viscosity. The value of $k_{c}$ for these phases is adopted from YLD04. For the WIM, the turbulent damping arises mainly from ion viscosity and collisionless damping. We calculate $k_{c}$ by equating the damping rate to the rate of turbulence cascade (see Appendix C, also YL03; YLD04).

Figure 4 sketches possible acceleration mechanisms present in MHD turbulence. Working scales in the inertial range, spanning from the injection scale $k_{\min }$ to the cutoff scale $k_{c}$ of turbulence, are indicated. Gyroresonance works only if $k_{\text {res }} \leq k_{c}$, and is dominant at small scales $k \geq k_{\text {res }} \sim r_{g}^{-1}$. The critical size $a_{\text {cri }}$ for gyroresonance is then obtained by solving the equation $k_{\text {res }}=k_{c}$ for grain size.

Figure 5 shows the rate of energy gain (Eq. 14) as a function of grain velocity $v$ for gyroresonance acceleration $(n= \pm 1)$ and TTD acceleration $(n=0)$ in the CNM (upper panel) and WIM (lower panel) arising from fast modes in MHD turbulence. Here we assumed that the 

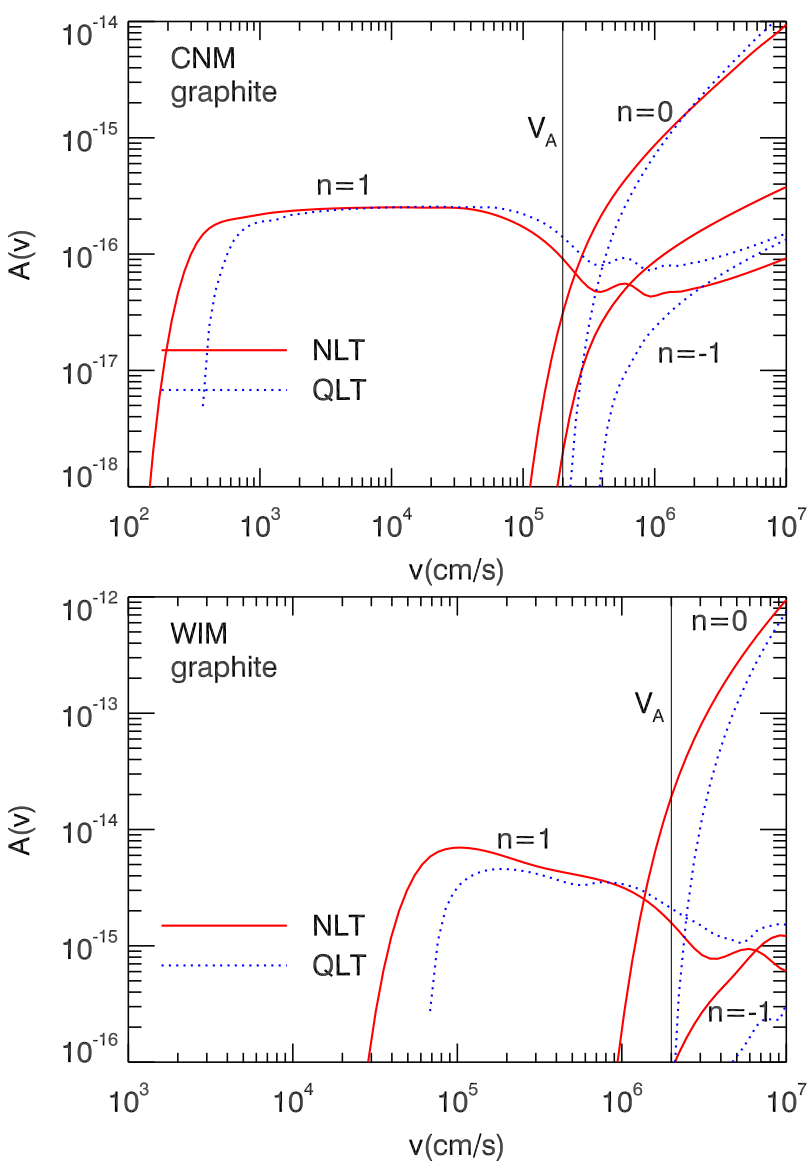

FIG. 5.- Rate of energy gain as a function of the grain velocity for gyroresonance acceleration $(n= \pm 1)$ and transit time damping acceleration ( $n=0$, TTD) for a graphite grain of size $a=10^{-5} \mathrm{~cm}$ in the CNM (upper) and WIM (lower). Solid and dot lines denote results from NLT and QLT, respectively. The Alfvénic speed $V_{\mathrm{A}}$ is indicated. The gyroresonance acceleration $(n=1)$ is dominant for $v<V_{\mathrm{A}}$, and the TTD acceleration $(n=0)$ takes over when $v \geq V_{\mathrm{A}}$. The case of efficient pitch angle scattering is considered.

pitch angle scattering is efficient. Due to the broadening of resonance condition, gyroresonant interactions occur at lower velocities in the NLT than QLT (solid lines). It can also be seen that the gyroresonance acceleration $(n=1)$ is dominant for $v<V_{\mathrm{A}}$, while TTD acceleration $(n=0)$ becomes dominant for $v \geq V_{\mathrm{A}}$.

In both the CNM and WIM, as $v \rightarrow V_{\mathrm{A}}, A_{v}(n=1)$ becomes smaller in the NLT than the QLT. As a result, we expect that gyroresonance acceleration is less efficient in the former case. We are going to quantify such a difference in the following.

\subsection{Gyroresonance in quasi-linear theory and nonlinear theory}

Grain velocities due to gyroresonance acceleration are obtained by solving Equation (13) using the diffusion coefficients from Equations (10) and (15). We assume that at the beginning the grain has low velocity, so that the pitch angle scattering by TTD is negligible. The gyroresonance increases rapidly $v_{\perp}$, and $\mu$ decreases to $\mu=0$. So, we can assume $\mu=0$ for the gyroresonance acceleration.

Figure 6 shows grain velocities obtained using the NLT and QLT for the CNM, WNM and WIM. Both silicate and graphite grains are considered. Grain velocities ob-
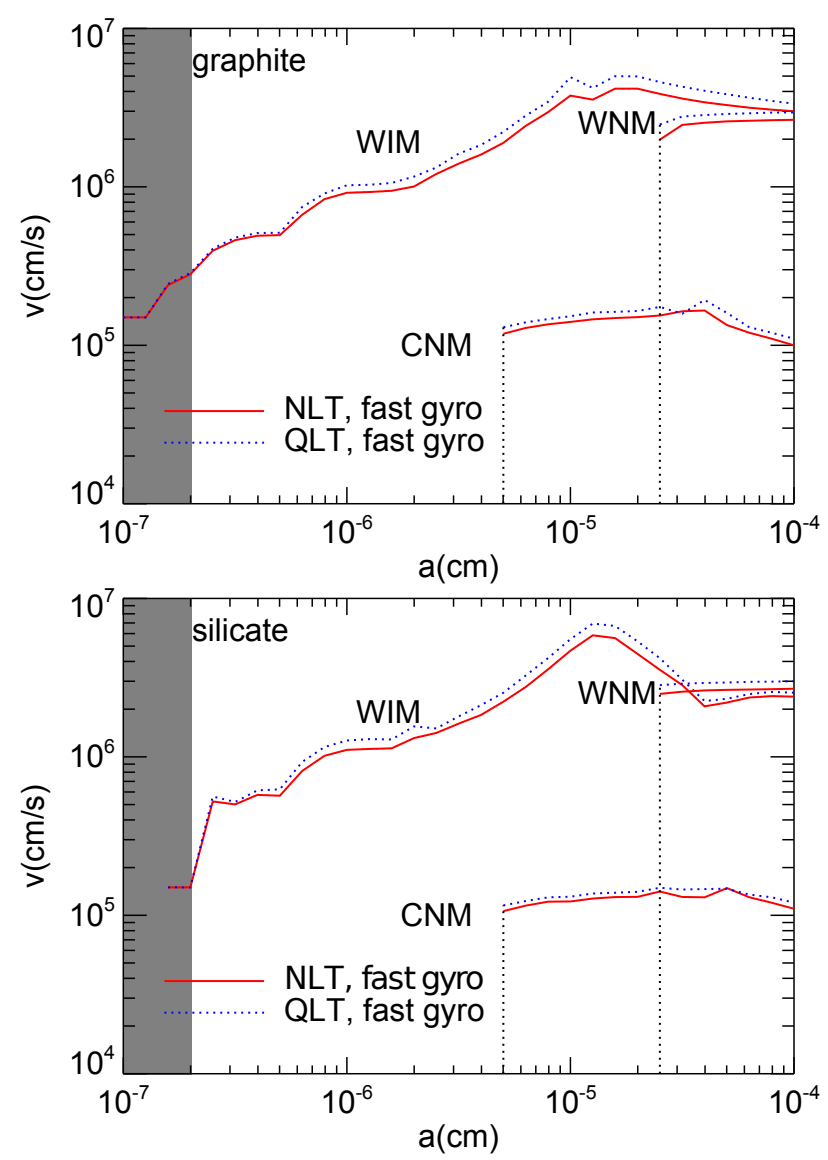

FIG. 6.- Grain velocities relative to gas as a function of grain size for graphite and silicate grains in various phases of the ISM. Acceleration arising from gyroresonant interactions of fast modes with grains are obtained using the QLT (dotted line) and NLT (solid line). The difference in velocities from the NLT and QLT is within $15 \%$. The shaded area indicates the range of size in which the assumption of constant charge is invalid due to strong charge fluctuations. Dotted vertical lines present in the WNM and CNM denote the critical size $a_{\text {cri }}$ corresponding to the cutoff scale $k_{c}$ of turbulence.

tained using the NLT are generically smaller than those from the QLT. But the difference is within $15 \%$. The smaller results in the NLT arise from the fact that, when grain velocities approach $V_{\mathrm{A}}$, a fraction of turbulence energy is spent to induce transit time acceleration (see also Fig. 5). The sudden cutoffs (dotted lines) present in the CNM and WNM correspond to the cutoff scales of turbulence due to collisional and collisionless damping occurring at the critical size $a_{\text {cri }}$ (see Sec. 4.1).

Figures 7 and 8 compare grain velocities arising from fast MHD modes (similar data as Fig. 6) with those induced by Alfvén hydro-drag modes (LY02) and fast hydro-drag modes (YLD04) in the CNM and WIM, respectively. ${ }^{3}$ As expected from earlier studies, gyroresonance acceleration is dominant for the entire range of grain size in the WIM (Fig. VNQwim). The rapid variation of grain mean charge $\langle Z\rangle$ present in the WIM (see Fig. 1) results in the non monotonic increase of grain velocities from gyroresonance (solid and dotted lines). Two local maxima in the hydro drag cases correspond to the change in sign of $\langle Z\rangle$ (see Fig. 1). In the CNM, gyroreso-

${ }^{3}$ Here only the velocity component perpendicular to magnetic field is shown. 

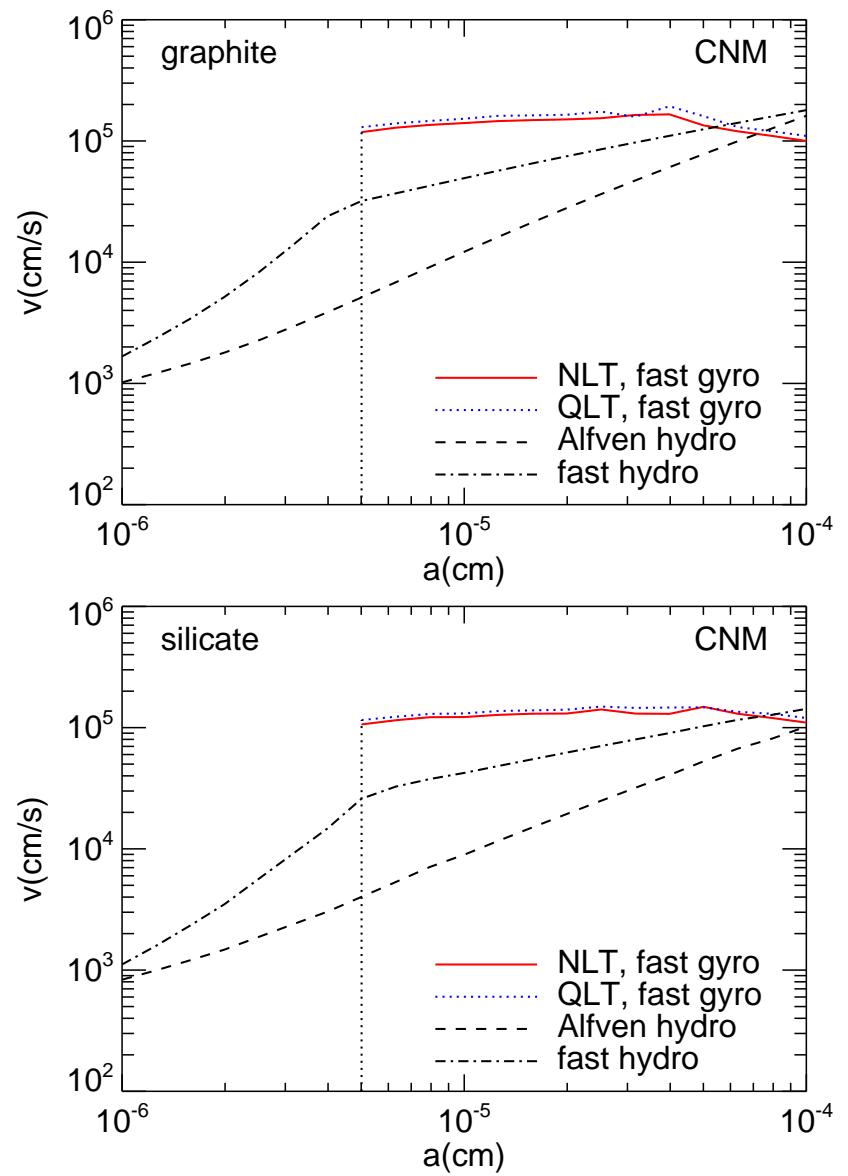

FIG. 7.- Comparison of grain velocities arising from gyroresonant interactions of fast MHD modes (solid and dotted lines) with the results arising from hydrodrag by fast and Alfvén modes (dotdashed and dashed lines).

nance is dominant for grain size from $a_{\text {cri }}=5 \times 10^{-6} \mathrm{~cm}$ to $a \sim 6 \times 10^{-5} \mathrm{~cm}$, while acceleration by hydro-drag takes over for grains smaller than $a_{\text {cri }}$ and larger than $\sim 6 \times 10^{-5} \mathrm{~cm}$ (see Fig. 7).

\subsection{Acceleration by TTD}

Gyroresonant acceleration tends to drive grain motion in perpendicular direction to the mean magnetic field (i.e., $\mu=0$ ). As discussed in Section 3.2, the broadening of resonance condition in the NLT allows TTD to operate even at $\mu=0$. In addition to acceleration, the scattering by TTD can be important, which results in the deviation of $\mu$ from $\mu=0$ (see Yan \& Lazarian 2008). However, it is still uncertain how fast the pitch angle scattering (both gyroresonance and TTD) by fast MHD modes is compared to the acceleration. For simplicity, we consider the TTD acceleration for two limiting cases of efficient scattering and inefficient scattering in which the scattering is more and less efficient than the acceleration, respectively. In the latter case, the scattering is assumed to be sufficient to alter the adiabatic invariant of gyromotion.

In the presence of TTD, we take into account the diffusion coefficients $D_{p p}^{\mathrm{TTD}}$ from Equation (12) for Equation (13) in addition to $D_{p p}^{\mathrm{G}}$.

When the pitch angle scattering is efficient, $\mu$ is described by an isotropic distribution $f(\mu) d \mu=1 / 2 d \mu$.
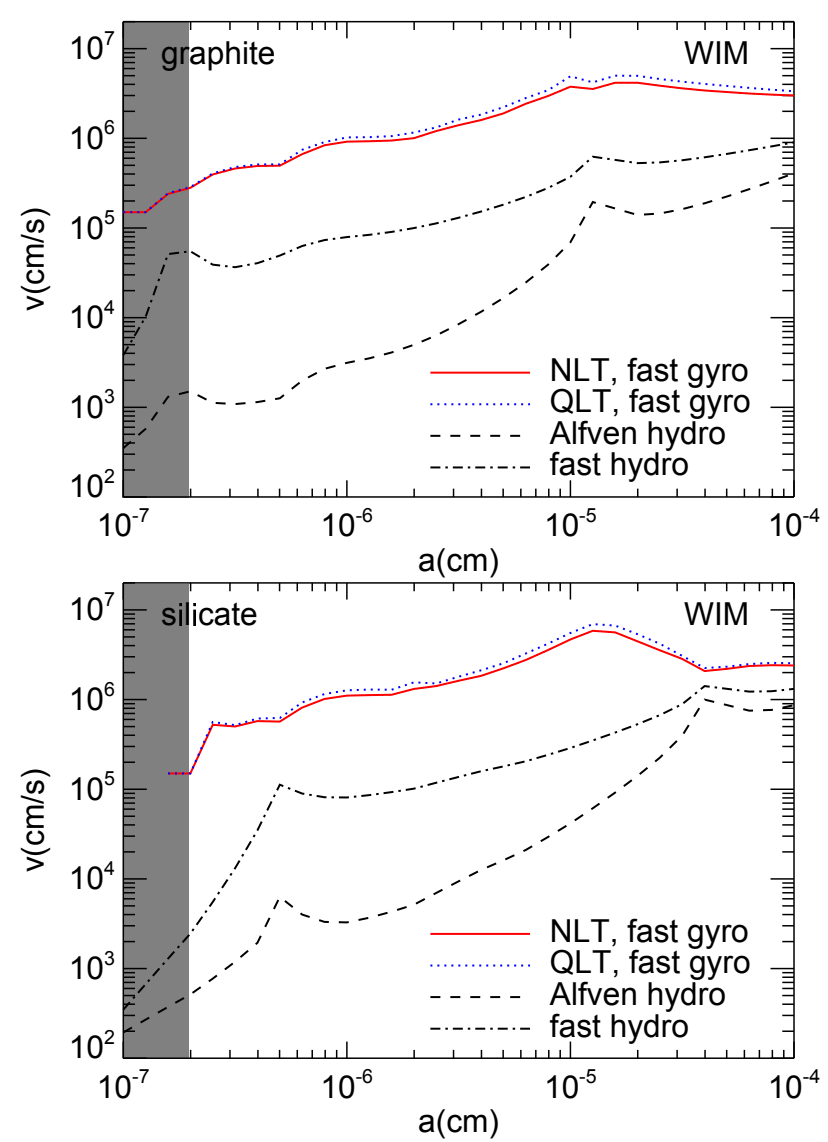

FIG. 8. - Similar to Fig. 7 but for the WIM. The shaded area indicates the range of grain size in which the assumption of constant charge is invalid. Local maximum velocity for hydro acceleration present in the range $a<10^{-6}$ and $a>10^{-5} \mathrm{~cm}$ arise from the change in sign of $\langle Z\rangle$.

The diffusion coefficient $D_{p}(p, \mu)$ is replaced by its average value over the isotropic distribution. $f(\mu)$.

Figure 9 compares grain velocities relative to gas obtained using the NLT from gyroresonance (dot line), and gyroresonance plus TTD by fast MHD modes for silicate grains (upper) and graphite grains (lower) assuming the efficient pitch angle scattering. In the WIM conditions, the TTD acceleration is negligible for grains smaller than $\sim 4 \times 10^{-6} \mathrm{~cm}$ for which $v<V_{\mathrm{A}}$. The efficiency of TTD begins to increase rapidly with $a$ when $v \sim V_{\mathrm{A}}$. TTD can increase grain velocities to an order of magnitude higher than gyroresonance. The effect of TTD is less important in the WNM than the WIM, but still considerable. For the CNM, TTD acceleration is rather marginal because grains are moving with sub-Alfvénic velocities, less than the threshold for TTD.

In the case of inefficient pitch angle scattering, the cosine of the grain pitch angle $\mu=0$ is assumed as a result of gyroresonance acceleration. We found that grain velocities are $\sim 10 \%$ larger than the results for the case of efficient scattering. This seems counterintuitive because the scattering is required to alter the adiabatic invariant in gyromotion. However, here we neglected that effect of pitch angle scattering, and the situation is merely related to the fact that the diffusion coefficient $D_{p p}$ averaged over $\mu$ is slightly lower than $D_{p p}$ at $\mu=0$ (see Fig. 3). 

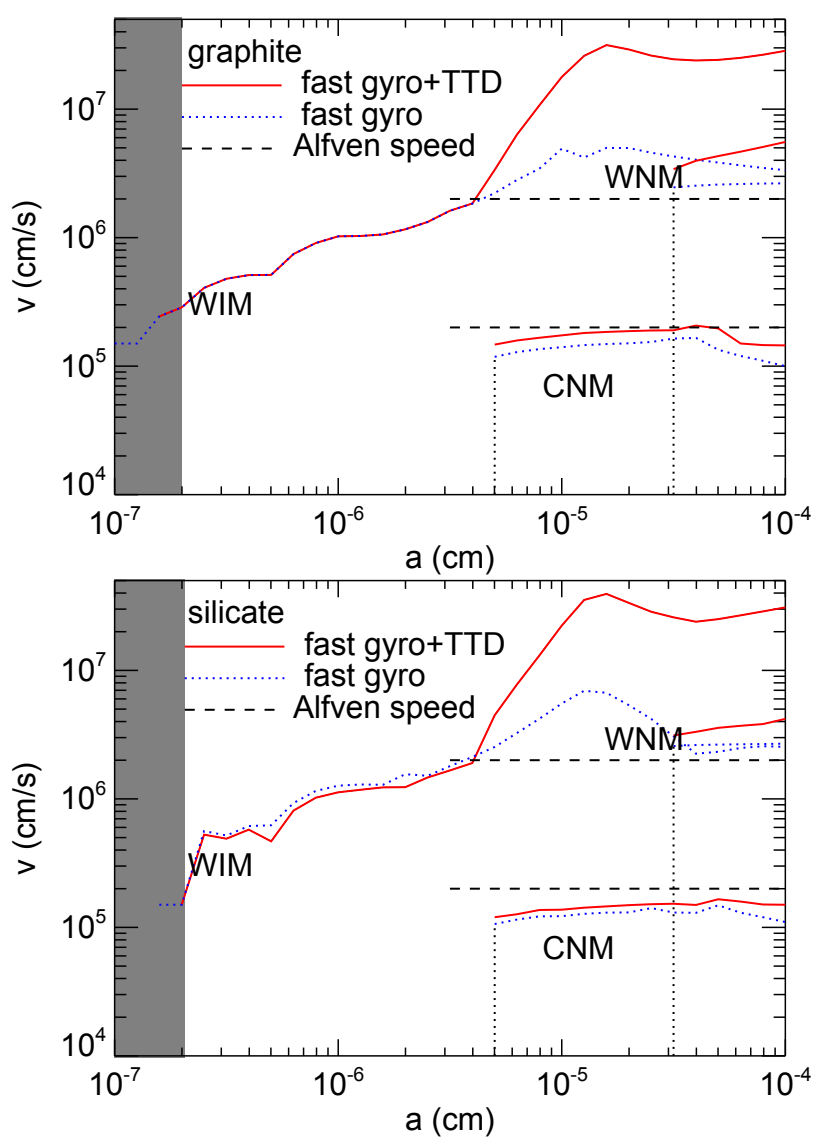

FIG. 9.- Grain velocities relative to gas arising from gyroresonant acceleration (dotted line) and gyroresonant acceleration plus transit time acceleration (solid line), as a function of grain size for different environments. The case of efficient scattering is considered.

\section{STOCHASTIC ACCELERATION BY LOW FREQUENCY ALFVÉN WAVES}

\subsection{General consideration}

The stochastic acceleration by low frequency Alfvén waves with $\omega<\Omega$ at the gyro-scale $k_{\perp} \sim \rho^{-1}$ was studied in Chandran et al. (2010) for ion heating in the solar wind. Here we consider the effect of low frequency Alfvén waves on dust acceleration in the ISM. For the low frequency Alfvén waves, resonance acceleration is inefficient because the resonance condition $\omega-k_{\|} v \mu=n \Omega$ is not satisfied. Indeed, in low- $\beta$ plasma, the ion thermal velocity $v_{T} \ll V_{\mathrm{A}}$, and $\omega=k_{\|} V_{\mathrm{A}}$ for Alfvén waves, we have $\omega-k_{\|} v \mu \ll 0$.

Let $\delta v$ and $\delta B$ be the rms amplitudes of velocity and magnetic field at the gyro-scale $k_{\perp} \rho \sim 1$. The electric field induced by plasma perturbations with velocity $\delta v$ in the direction perpendicular to the mean magnetic field has the rms amplitude

$$
\delta E \simeq \frac{\delta v B_{0}}{c},
$$

and the potential is written as

$$
\delta \Phi \simeq \rho \delta E .
$$

The electric field in the plane perpendicular to $\mathbf{B}_{0}$ results in the acceleration of grains in the perpendicular direction.
Combining these above equations, we obtain

$$
q \delta \Phi \simeq q \frac{\rho \delta v B_{0}}{c}=m v_{\perp} \delta v,
$$

where $m$ is mass of the charged particle and $\rho=$ $m c v_{\perp} / q B_{0}$ is the gyro radius.

The fractional increase of energy after a single gyroperiod is calculated by

$$
\frac{q \delta \Phi}{m v_{\perp}^{2} / 2} \simeq 2 \epsilon
$$

where

$$
\epsilon=\frac{\delta v}{v_{\perp}},
$$

When $\epsilon \ll 1$, also corresponding to $\delta B \ll B_{0}$ due to the assumption of low $\beta$ plasma, the increase of energy per gyroperiod is negligible because of adiabatic invariant for the magnetic moment $m v_{\perp}^{2} / 2 B_{0}$. As $\epsilon$ increases to unity, the particle energy changes substantially after one gyroperiod. The guiding center becomes chaotic when $\epsilon$ exceeds some threshold value, so that the perpendicular acceleration becomes important.

The increase of energy depends only on the amplitude of perturbation at the scale of gyroradius. The increase of energy per unit of time per unit of mass is defined as

$$
Q_{\perp}=\frac{v_{\perp}^{2}}{\tau_{\text {acc }}},
$$

where $\tau_{\text {acc }}$ is the time for the particle kinetic energy $K_{\perp}$ to increases by a factor of 2 . We can estimate $\tau_{\text {acc }}$ using the diffusion coefficient $D_{K}$ as follows:

$$
\tau_{\text {acc }}=\frac{4 K_{\perp}^{2}}{D_{K}}=\frac{m^{2} v_{\perp}^{4}}{D_{K}},
$$

where

$$
D_{K}=\frac{\left(\Delta K_{\perp}\right)^{2}}{\Delta t}=m^{2} v_{\perp}^{2} \omega_{\text {eff }}^{2} \delta v \rho,
$$

and $\omega_{\text {eff }}=\delta v / \rho$ is the effective frequency of gyroscale fluctuations. Here we have taken $\Delta K_{\perp} \simeq m v_{\perp} \delta v=$ $m v_{\perp} \omega_{\text {eff }} \rho$ from Equation (18), and $\Delta t=\rho / \delta v$ is the time required for the guiding center to move by a distance equal to the gyro radius $\rho$.

Taking use of Equations (22) and (23) for (21), we obtain

$$
Q_{\perp} \simeq \omega_{\mathrm{eff}}^{2} \delta v \rho \simeq \frac{\delta v^{3}}{\rho}
$$

When $\epsilon$ is sufficiently small, the variation in the particle energy is correlated over long time, so the assumption for diffusive approximation may not be adequate. Thus, the actual energy gain is substantially smaller than the value obtained by Equation (24). To account for this reduction, a damping function $\exp \left(-c_{2} / \epsilon\right)$ is introduced, and Equation (24) can be rewritten as

$$
Q_{\perp}=\frac{c_{1}(\delta v)^{3}}{\rho} \exp \left(-\frac{c_{2}}{\epsilon}\right),
$$

where $c_{1}$ and $c_{2}$ are dimensionless constants that depend on the nature of fluctuations. Below we assume $c_{1}=$ 
0.75 and $c_{2}=0.34$ for the fluctuations by low frequency Alfvén waves as in Chandran et al. (2010).

\subsection{Grain velocities for the ISM}

Let assume that the Alfvénic turbulence in the ISM follows the scaling

$$
\delta v_{\perp}=\alpha V_{\mathrm{A}}\left(\frac{l_{\perp}}{L}\right)^{a}
$$

where $l_{\perp} \sim \rho_{\mathrm{d}}$ and $L$ are gyro scale and the injection scale. $\alpha$ and $a=\left(c_{3}-1\right) / 2$ with $c_{3}$ being the slope of turbulence power spectrum are dimensionless, derived from the properties of turbulence. For sub-Alfvénic turbulence, $\alpha<1$. Using Equation (20) for dust grains, i.e., $\epsilon_{\mathrm{d}}=\delta v_{\perp} / v_{\perp, d}$, we obtain

$$
\epsilon_{d}=\alpha\left(\frac{B^{2}}{8 \pi n_{\mathrm{H}} k_{\mathrm{B}} T_{\perp}}\right)^{(1-a) / 2} \frac{A^{(1+a) / 2}}{Z^{a}}\left(\frac{d_{p}}{L}\right)^{a},
$$

where $p$ denotes proton, and $d$ denotes dust, $A=m / m_{p}$, $d_{p}=V_{\mathrm{A}} / \Omega_{p}$, and the perpendicular temperature is defined as $k T_{\perp}=m v_{\perp, \mathrm{d}}^{2} / 2$.

Using Equations (26) and (27) for Equation (25), we obtain the rate of energy gain per a grain of mass $m$

$$
A\left(v_{\perp}\right)=m \frac{c_{1} V_{\mathrm{A}}^{3}}{L} \exp \left(-\frac{c_{2}}{\epsilon_{d}}\right) .
$$

For calculations, we assume $c_{3}=5 / 3$ and $a=1 / 3$ for Alfvénic turbulence above the gyro scale.

Using the parameters for the ISM in Table 1, we calculate the grain velocity arising from the chaotic acceleration by low frequency Alfvén waves in Figure 10 for the CNM and WIM. We show that the chaotic acceleration by low frequency Alfvén waves is subdominant to the fast and Alfvénic hydrodynamic drag. Obviously, it is much less important than gyroresonance and TTD by fast modes. The possible reason is that the low frequency Alfvén waves cascade faster to small scale than the fast modes.

\section{DISCUSSION}

\subsection{Related works on dust grain acceleration}

The acceleration of dust grains by incompressible MHD turbulence was first studied by Lazarian \& Yan (2002). Yan \& Lazarian (2003) studied grain acceleration in compressible MHD turbulence, and discovered a new acceleration mechanism based on gyroresonant interactions of grains with waves. This acceleration mechanism increases grain velocities in perpendicular direction to the mean magnetic field. YLD04 computed grain velocities arising from gyroresonance by fast MHD modes using quasi-linear theory (QLT), and compared the obtained results with different mechanisms, for various ISM phases. They found that the gyroresonance is the most efficient mechanism for grain acceleration in the ISM.

The effect of large scale compression on grain acceleration is shown by Yan (2009) to be less important than the gyroresonance in the ISM conditions, unless the grains move with super-Alfvénic velocities.

For very small grains (e.g., polycyclic aromatic hydrocarbons and nanoparticles), Ivlev et al. (2010) sketched
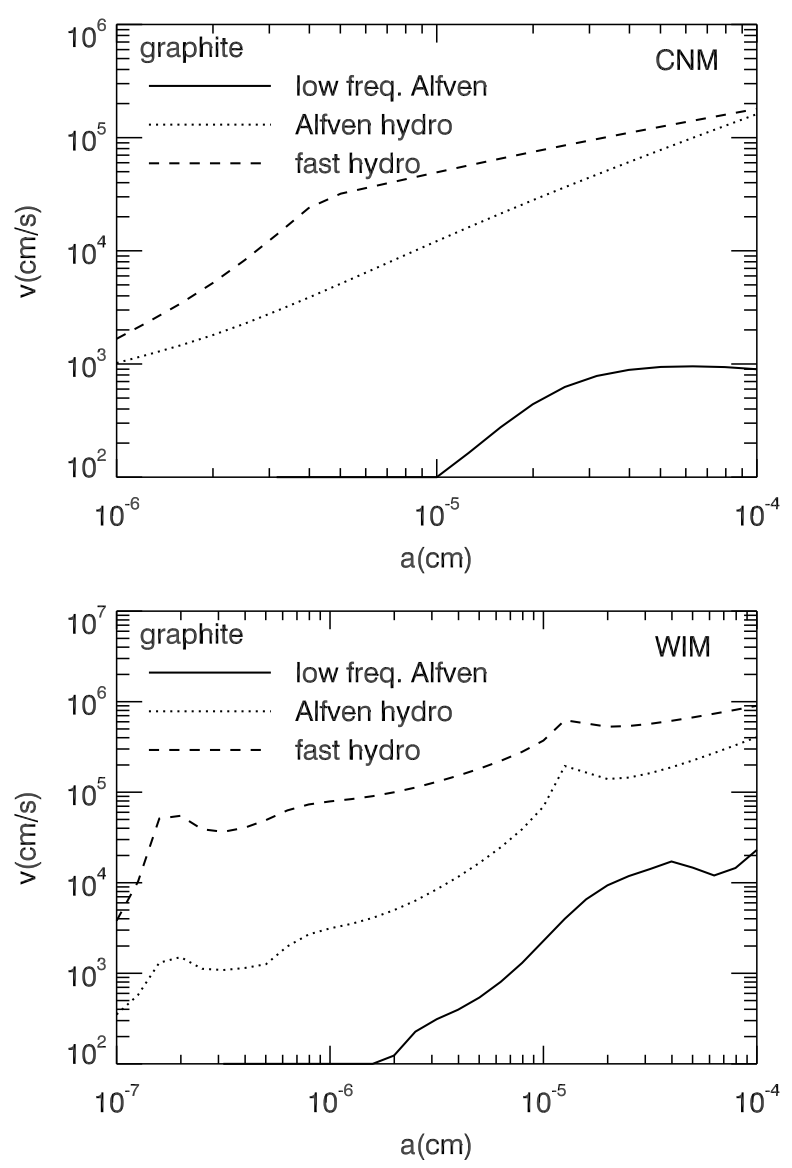

FIG. 10.- Grain velocity due to stochastic acceleration by low frequency Alfvén waves (solid lines) compared to the acceleration by hydrodynamic drag from Alfvén modes (dotted lines) and fast modes (dashed lines), for the CNM (upper) and WIM (lower). The stochastic acceleration is much less efficient than the latter.

a new mechanism of grain acceleration due to electrostatic interactions of grains with fluctuating charge and provided rough estimates of grain velocities in the ISM. Hoang \& Lazarian (2011) quantified this mechanism using Monte Carlo simulations of charge fluctuations. They found that charge fluctuations can accelerate grains to several times their thermal velocities.

\subsection{NLT for gyroresonance acceleration}

We have revisited the treatment of gyroresonance acceleration for charged grains due to MHD turbulence by accounting for the fluctuations of grain guiding center from a regular trajectory along the mean magnetic field (i.e. NLT limit). The fluctuations of the guiding center result in the broadening of resonance conditions- a Delta function is replaced by a Gaussian function. Such broadening of resonance condition allows some fraction of wave energy spent through the TTD acceleration. As a result, grain velocities due to gyroresonance acceleration are in general decreased by $\sim 15 \%$ in the NLT limit.

\subsection{Transit time damping acceleration}

TTD acceleration is believed to be important when the parallel component of grain velocity along the magnetic field exceeds the Alfvén speed $V_{\mathrm{A}}$. Although gyroresonance acceleration by fast modes can accelerate grains to $v \geq V_{\mathrm{A}}$, their resulting velocity mostly perpendicular to 
the magnetic field, i.e. $\mu=0$, makes TTD unfavored because the resonance condition $\delta\left(\omega-k_{\|} v_{\|}\right)$is not satisfied. Indeed, we found that TTD is efficient for $\mu>V_{\mathrm{A}} / v$ and negligible for $\mu<V_{\mathrm{A}} / v$ in the QLT limit. This feature is consistent with the result for acceleration of cosmic rays in Schlickeiser \& Miller (1998).

The situation changes when the fluctuations of the guiding center are taken into account in the NLT. For this case, the resonance condition is broadened beyond the $\delta$ function, and can be described by a Gaussian function. As a result, TTD acceleration becomes important for $\mu<V_{\mathrm{A}} / v$, including $90^{\circ}$ pitch angle.

In addition to acceleration, TTD also induces the grain pitch angle scattering, which is dominant over the scattering by gyroresonance. Since the efficiency of the TTD scattering is uncertain, we considered in the paper two limiting cases of inefficient and efficient scattering in which the scattering is less and more efficient than the acceleration. The pitch angle is equal to $90^{\circ}$ in the former, and isotropic in the latter.

When the scattering is more efficient than the acceleration, we showed that for the WNM and WIM, the TTD acceleration can increase substantially the grain velocity compared to results arising from gyroresonance. Particularly, for grains larger than $5 \times 10^{-6} \mathrm{~cm}$ in the WIM, TTD acceleration is an order of magnitude greater than the gyroresonance acceleration. TTD is clearly more efficient than the betatron acceleration studied in Yan (2009). In the CNM, TTD acceleration is limited because the gyroresonance acceleration is not able to speed up grains to super Alfvénic stage. When the scattering is less efficient than the acceleration, grain velocities are within $10 \%$ lower than the results for the efficient scattering case.

\subsection{Stochastic acceleration}

We study also the effect of low frequency $(\omega<\Omega)$ Alfvén waves on dust grains in the ISM. We show that the stochastic acceleration by low frequency Alfvén waves is subdominant to the gyro-resonance acceleration and TTD acceleration by fast modes. This may arise from the fact that low frequency Alfvén waves cascade faster to small scale than the fast modes in MHD turbulence.

\subsection{Implication to dust coagulation and shattering and alignment}

Hirashita \& Yan (2009) adopted grain velocity due to gyroresonance acceleration from YLD04 to model grain size distribution in the different ISM conditions. Hirashita et al. (2010) studied grain coagulation and shattering in the WIM for large dust grains ejected from Type II supernova. They showed that the shattering of large dust grains due to turbulence plays an important role in producing small size population that modifies extinction curves in starburst galaxies.

The threshold velocity for the grain shattering is a function of the grain size:

$$
v_{\text {shat }}=2.7\left(\frac{a}{10^{-7} \mathrm{~cm}}\right)^{-5 / 6} \mathrm{~km} \mathrm{~s}^{-1} \text {, }
$$

where $v_{\mathrm{dd}}$ is the relative velocity of dust grains (Chokshi et al. 1993). If $v_{\text {dd }}<v_{\text {shat }}$, the grains collide and stick together. When $v_{\text {dd }}>v_{\text {shat }}$, the collisions with high velocity produce shock waves inside the grains, and shatter them in smaller fragments. For $v_{\mathrm{dd}} \geq 20 \mathrm{~km} / \mathrm{s}$, the evaporation of the dust grain occurs and the grains are destroyed.

When TTD is accounted for, grains larger than $5 \times 10^{-6}$ in the WIM and WNM may undergo efficient shattering because $v_{\mathrm{dd}}>V_{\mathrm{A}}=20 \mathrm{~km} \mathrm{~s}^{-1}$.

The effects of high velocities we obtain on dust grain alignment require further studies. Recent research has shown that the classical grain alignment theory of Davis \& Greenstein (1951) (see also Lazarian 1995; Roberge \& Lazarian 1999 for more recent quantitative studies of the process) is subdominant to the radiative torque (RAT) model (see Dolginov \& Mitrofanov 1976; Draine \& Weingartner 1996; Lazarian \& Hoang 2007a). This model, however, was criticized in Jordan \& Weingartner (2009) who appealed to the results of gyroresonance acceleration of grains in Yan \& Lazarian (2003) and claimed this means that fast moving grains will be randomized as their charge fluctuates. Our results indicate that grains can be accelerated to even faster velocities, which could make the problem for the alignment more severe. However, we believe that the claim about suppression of the RAT alignment for fast moving grains is a result of the confusion on the nature of the RAT alignment. We plan to address this issue elsewhere. At the same time high velocities of grains may induce another mechanical alignment of irregular grains as it described in Lazarian \& Hoang (2007b). This alignment does require further studies.

\section{SUMMARY}

In the present paper, we study the resonance acceleration of charged grains by fast modes and stochastic acceleration by Alfvén waves in MHD turbulence. Our main results are summarized as follows.

1. We revisit the treatment of gyroresonance acceleration of charged grains in compressible MHD turbulence by taking into account the fluctuations of grain guiding center from the regular trajectory along the mean field. Gyroresonance interactions by fast modes can accelerate large grains to super-Alfvénic speed. We found that grain velocities are lower by $15 \%$ in the NLT than the QLT.

2. We investigate the effect of transit time damping (TTD) by fast modes for super-Alfvénic grains. We found that the fluctuations of grain guiding center allow TTD to occur not only within the range of the cosine of the grain pitch angle $\mu>V_{\mathrm{A}} / v$ as expected by the QLT, but also for $\mu<V_{\mathrm{A}} / v$. We show that the TTD acceleration can increase grain velocities by an order of magnitude compared to the results arising from gyroresonance mechanism Thus, TTD is the most efficient acceleration mechanism for super-Alfvénic grains.

3. The stochastic acceleration due to low frequency Alfvén waves is inefficient for dust grains in the ISM conditions.

AL thanks Alexander von Humboldt Foundation. R.S. acknowledges the support from the Deutsche Forschungsgemeinschaft through grants Schl 201/19-1 and Schl 201/23-1. TH and AL acknowledge the support of the Center for Magnetic Self-Organization. We thank Bruce 
Draine for providing us the data of grain charge distribution and Huirong Yan for valuable comments. We thank the anonymous referee for her/his useful comments that improve the paper.

\section{APPENDIX}

\section{A. TURBULENCE CASCADE AND DAMPING}

We summarize here major damping processes for the MHD turbulence. First, we begin with the cascade of different MHD turbulence modes.

\section{A.1. Turbulence cascade}

Turbulence cascades from large scale to small scale. The cascade rate depends on the scale of the eddy and on the type of turbulence mode. For Alfvén and pseudo-Alfvén (slow) modes, the cascade time at the scale $k$ is equal to the eddy turn-over time:

$$
\tau_{\text {cas }}^{A, s}=\frac{1}{k_{\|} V_{\mathrm{A}}} \equiv \frac{1}{k_{\perp} v_{k}} .
$$

where the critical balance condition (GS95) for the cascade parallel and perpendicular to the mean magnetic field

$$
k_{\|} v_{\mathrm{A}}=k_{\perp} v_{\perp}
$$

has been used. Here $v_{\perp} \sim v_{k}$ because the Alfvén mode has perturbation velocity $v_{k}$ perpendicular to the mean magnetic field.

Fast modes cascade a bit slower than the eddy turn over, and the cascade time is given by

$$
\tau_{\text {cas }}^{f}=\left(\frac{l}{v_{k}}\right)\left(\frac{v_{p h}}{v_{k}}\right)=\frac{k^{-1} V_{f}}{v_{k}^{2}} \sim\left(\frac{k}{L}\right)^{1 / 2} \frac{\delta V^{2}}{v_{p h}},
$$

where $v_{p h}$ is the phase speed. In low- $\beta$ plasma, $v_{p h} \equiv v_{\mathrm{A}}$ and $v_{p h} \equiv c_{s}$ in high- $\beta$ plasma.

\section{B. TURBULENCE DAMPING}

\section{B.1. Collisional damping}

\section{B.1.1. Neutral Ion collision damping}

In partially ionized gas, the MHD turbulence energy is dissipated through ion-neutral collisions. Let $\lambda_{n}$ be the mean free path of a neutral. For scales $l>\lambda_{n}$, the ion-neutral collisions can damp the turbulence at a damping rate

$$
\Gamma_{n i} \sim \nu_{n} l^{-2} \sim \frac{n_{n}}{n_{\text {gas }}}\left(\lambda_{n} v_{n}\right) k^{2}
$$

where $l \sim k^{-1}$ is the scale of interest, $\nu_{n}$ is the effective viscosity due to neutrals, and $n_{\text {gas }}=n_{i}+n_{n}$ is the gas number density.

\section{B.1.2. Viscous damping}

In fully ionized gas, the turbulence can get damped due to viscous damping or collisionless damping depending on whether regimes are collisional or collisionless. The Coulomb mean free path of the thermal plasma is given by

$$
\lambda_{\text {Coul }}=v \tau_{\text {Coul }}=\frac{v}{n v \sigma}=\frac{m^{2} v^{4}}{n \pi e^{4}}=9 \times 10^{7}\left(\frac{T_{\text {gas }}}{10^{7} \mathrm{~K}}\right)^{2}\left(\frac{10^{10} \mathrm{~cm}^{-3}}{n}\right) \mathrm{cm},
$$

where $\sigma=\pi r_{\min }^{2}=\pi\left(e^{2} / m v^{2}\right)$ has been used.

The viscous damping is important for scales between the injection scale $L$ and the mean free path $\lambda_{\text {Coul }}$. For scales $l<\lambda_{\text {Coul }}$, the collisionless damping is dominant.

\section{B.2. Collisionless regime \\ B.2.1. ion viscosity damping}

The motion in the perpendicular direction to the magnetic field is suppressed, so the viscosity is much smaller in the perpendicular direction compared to the viscosity in the parallel direction, i.e. $\eta_{\perp} \sim \eta_{0} /\left(\Omega_{i} \tau_{\text {Coul }}\right)^{2}$ where $\tau_{\text {Coul }} \sim \lambda_{\text {Coul }} / v_{t h}$ is the Coulomb collision time for ion and $v_{t h}$ is the ion thermal velocity, and $\eta_{0}=0.96 n k_{B} T \tau_{\text {Coul }}$ is the longitudinal viscosity for motion along the magnetic field (see Braginskii 1965). Petrosian et al. (2006) derived

$$
\Gamma_{i v i s c}=k_{\perp}^{2} \frac{\eta_{0}}{6 n_{i} m_{i}},
$$

for $\beta \ll 1$ and

$$
\Gamma_{i v i s c}=\frac{k^{2} \eta_{0}\left(1-3 \cos \theta^{2}\right)^{2}}{6 n_{i} m_{i}}
$$


for $\beta \gg 1$. For fast modes, the cut-off due to viscous damping is obtained in YL08:

$$
k_{c} L=x_{c}\left(1-\cos \beta^{2}\right)^{-2 / 3}
$$

for $\beta \ll 1$ and

$$
k_{c} L=x_{c}\left(1-3 \cos \beta^{2}\right)^{-4 / 3}
$$

for the high $\beta$ plasma, where $x_{c}=\left(6 \rho \delta V^{2} L /\left(\eta_{0} V_{\mathrm{A}}\right)\right)^{2 / 3}$.

For fast modes, the cut-off scale of turbulence is given by

$$
k_{c} L=\left(\frac{L \beta}{18}\right)^{-1 / 3}\left(\frac{\lambda_{\text {Coul }} \sin \theta^{2}}{M_{\mathrm{A}}^{2}}\right)^{-2 / 3}=\frac{4}{\beta^{1 / 3}}\left(\frac{M_{\mathrm{A}}}{\sin \theta}\right)^{4 / 3}\left(\frac{L}{10^{8} \mathrm{~cm}}\right)^{2 / 3}\left(\frac{10^{10} \mathrm{~cm}}{n}\right)\left(\frac{10^{7} \mathrm{~K}}{T_{\text {gas }}}\right) .
$$

\section{B.2.2. Landau damping}

In collisionless plasma, the turbulence is damped due to the Landau damping. The damping rate due to the Landau damping is given by (see YL08)

$$
\Gamma_{n c o l}=\frac{\sqrt{\pi \beta} \sin \theta^{2}}{2 \cos \theta} k v_{\mathrm{A}}\left[\left(\frac{m_{e}}{m_{i}}\right)^{1 / 2} \exp -\left(\frac{m_{e}}{\beta m_{i} \cos \theta^{2}}\right)+5 \exp -\left(\frac{1}{\beta \cos \theta^{2}}\right)\right] .
$$

The cut-off scale of the turbulence is obtained by equating the damping rate to the cascading rate. From Equations (A3) and (B8) we obtain

$$
k_{c} L=\frac{4 M_{\mathrm{A}}^{2} m_{i} \cos \theta^{2}}{\pi m_{e} \beta \sin \theta^{4}} \exp \left(-\frac{2 m_{e}}{\beta m_{i} \cos \theta^{2}}\right)
$$

\section{NLT OF GYRORESONANCE ACCELERATION OF DUST GRAINS}

Below we describe the NLT for resonance acceleration of dust grains in MHD turbulence.

\section{C.1. Fokker-Plank coefficients}

The QLT assumes that the guiding center of the charged particles is regular motion along the uniform magnetic field and that the gyro-orbit is not perturbed.

The Fokker-Planck diffusion coefficients (Jokipii 1966; Schlickeiser \& Miller 1998) are given as

$$
\begin{aligned}
\left(\begin{array}{c}
D_{\mu \mu} \\
D_{p p}
\end{array}\right)= & \frac{\pi \Omega^{2}\left(1-\mu^{2}\right) \delta V^{2}}{u_{B}} \int_{\mathbf{k}_{\text {min }}}^{\mathbf{k}_{\max }} d k^{3} R_{n}\left(k_{\|} v \mu-\omega+n \Omega\right)\left(\left(\begin{array}{c}
\left.1+\frac{\mu V_{p h}}{v \zeta}\right)^{2} \\
m^{2} V_{A}^{2}
\end{array}\right)\right. \\
& \left\{\left(J_{2}^{2}\left(\frac{k_{\perp} v_{\perp}}{\Omega}\right)+J_{0}^{2}\left(\frac{k_{\perp} v_{\perp}}{\Omega}\right)\right)\left[\begin{array}{c}
M_{\mathcal{R} \mathcal{R}}(\mathbf{k})+M_{\mathcal{L} \mathcal{L}}(\mathbf{k}) \\
K_{\mathcal{R} \mathcal{R}}(\mathbf{k})+K_{\mathcal{L} \mathcal{L}}(\mathbf{k})
\end{array}\right]\right. \\
- & \left.2 J_{2}\left(\frac{k_{\perp} v_{\perp}}{\Omega}\right) J_{0}\left(\frac{k_{\perp} v_{\perp}}{\Omega}\right)\left[e^{i 2 \phi}\left[\begin{array}{c}
M_{\mathcal{R} \mathcal{L}}(\mathbf{k}) \\
K_{\mathcal{R} \mathcal{L}}(\mathbf{k})
\end{array}\right]+e^{-i 2 \phi}\left[\begin{array}{c}
M_{\mathcal{L} \mathcal{R}}(\mathbf{k}) \\
K_{\mathcal{L R}}(\mathbf{k})
\end{array}\right]\right]\right\},
\end{aligned}
$$

where $u_{B}=B_{0}^{2} / 8 \pi,\left|\mathbf{k}_{\min }\right|=k_{\min }=L^{-1},\left|\mathbf{k}_{\max }\right|=k_{\max }$ corresponds to the dissipation scale, $\mathcal{R}, \mathcal{L}$ refer to the leftand right-circularly polarized modes, and $\phi=\tan ^{-1} k_{x} / k_{y}$. Above $R_{n}$ is the function for resonance condition, $V_{p h}$ is the phase speed and $\Omega$ is Larmor frequency.

The correlation tensors are defined as

$$
\begin{gathered}
\left\langle B_{\alpha}(\mathbf{k}, t) B_{\beta}^{*}\left(\mathbf{k}^{\prime}, t+\tau\right)\right\rangle / B_{0}^{2}=\delta\left(\mathbf{k}-\mathbf{k}^{\prime}\right) M_{\alpha \beta}(\mathbf{k}) e^{-\tau / \tau_{k}} \\
\left\langle v_{\alpha}(\mathbf{k}, t) v_{\beta}^{*}\left(\mathbf{k}^{\prime}, t+\tau\right)\right\rangle / V_{A}^{2}=\delta\left(\mathbf{k}-\mathbf{k}^{\prime}\right) K_{\alpha \beta}(\mathbf{k}) e^{-\tau / \tau_{k}},
\end{gathered}
$$

where $B_{\alpha, \beta}, v_{\alpha, \beta}$ are respectively the magnetic and velocity perturbation associated with the turbulence, $\tau_{k}$ is the nonlinear decorrelation time and essentially the cascading time of the turbulence. For the balanced cascade we consider (see discussion of our imbalanced cascade in CLV02), i.e., equal intensity of forward and backward waves, $C_{i j}(\mathbf{k})=0$.

The magnetic correlation tensor for Alfvénic turbulence is (CLV02),

$$
\begin{aligned}
{\left[\begin{array}{c}
M_{i j}(\mathbf{k}) \\
K_{i j}(\mathbf{k})
\end{array}\right] } & =\frac{L^{-1 / 3}}{12 \pi} I_{i j} k_{\perp}^{-10 / 3} \exp \left(-L^{1 / 3}\left|k_{\|}\right| / k_{\perp}^{2 / 3}\right), \\
\tau_{k} & =\left(L / V_{A}\right)\left(k_{\perp} L\right)^{-2 / 3} \sim\left(k_{\|} V_{A}\right)^{-1}
\end{aligned}
$$

where $I_{i j}=\left\{\delta_{i j}-k_{i} k_{j} / k^{2}\right\}$ is a $2 \mathrm{D}$ tensor in $x-y$ plane which is perpendicular to the magnetic field, $L$ is the injection scale, $V$ is the velocity at the injection scale. Slow modes are passive and similar to Alfvén modes. The 
normalization constant is obtained by assuming equipartition $\epsilon_{k}=\int d k^{3} \sum_{i=1}^{3} M_{i i} B_{0}^{2} / 8 \pi \sim B_{0}^{2} / 8 \pi$. The normalization for the following tensors below are obtained in the same way.

According to CL02, fast modes are isotropic and have one dimensional energy spectrum $E(k) \propto k^{-3 / 2}$. In low $\beta$ medium, the corresponding correlation is (YL03)

$$
\left[\begin{array}{c}
M_{i j}(\mathbf{k}) \\
K_{i j}(\mathbf{k})
\end{array}\right]=\frac{L^{-1 / 2}}{8 \pi} H_{i j} k^{-7 / 2}\left[\begin{array}{c}
\cos ^{2} \theta \\
1
\end{array}\right], \quad \tau_{k}=(k / L)^{-1 / 2} \times V_{A} / V^{2},
$$

where $\theta$ is the angle between $\mathbf{k}$ and $\mathbf{B}, H_{i j}=k_{i} k_{j} / k_{\perp}^{2}$ is also a $2 \mathrm{D}$ tensor in $x-y$ plane. The factor $\cos ^{2} \theta$ represents the projection as magnetic perturbation is perpendicular to $\mathbf{k}$. This tensor is different from that in Schlickeiser \& Miller (1998). For isotropic turbulence, the tensor of the form $\propto E_{k}\left(\delta_{i j}-k_{i} k_{j} / k^{2}\right)$ was obtained to satisfy the divergence free condition $\mathbf{k} \cdot \delta \mathbf{B}=0$ (see Schlickeiser 2002). Nevertheless, the fact that $\delta \mathbf{B}$ in fast modes is in the $\mathbf{k}$-B plane places another constraint on the tensor so that the term $\delta_{i j}$ doesn't exist.

\section{C.2. Diffusion coefficients in NLT}

The motion of a charged particle in a magnetic field $\mathbf{B}$ consists of the motion of the guiding center with respect to the magnetic field $\mathbf{B}$ and the motion of the particle about the guiding center. In the QLT limit, the guiding center is assumed to follow regular trajectory with constant pitch angle $\mu$. In MHD turbulence, $\mathbf{B}$ varies with respect to space and time, so $\mu$ changes, and $v_{\|}$and $v_{\perp}$ change accordingly.

In the NLT limit, the dispersion of the pitch angle due to magnetic field fluctuations reads

$$
\Delta \mu=\frac{\Delta v_{\|}}{v}
$$

and

$$
\frac{\Delta v_{\|}}{v_{\perp}}=\frac{\left\langle\left(B-B_{0}\right)^{2}\right\rangle^{1 / 4}}{B_{0}^{1 / 2}}=\left[\frac{\left\langle\left(\delta B_{\|}\right)^{2}\right\rangle}{B_{0}^{2}}+O \frac{\left\langle\left(\delta B_{\perp}\right)^{2}\right\rangle}{B_{0}^{2}}\right]^{1 / 4}
$$

where $B_{0}$ is the mean magnetic field (Völk 1975). The dispersion of parallel velocity $\delta v_{\|}$is mainly induced by the fluctuations of the parallel magnetic field $\delta B_{\|}$, while $\delta B_{\perp}$ is only second order effect.

Since $\mu$ is constant in the QLT, the resonance condition is given by $\delta\left(k_{\|} \mu v-\omega+n \Omega\right)$. In the NLT, due to the fluctuations of $\mu$ (Eq. C5), the resonance condition is broadened and described by

$$
R_{n}\left(k_{\|} v \mu-\omega+n \Omega\right)=\frac{\sqrt{\pi}}{k_{\|} \Delta v_{\|}} \exp \left[-\frac{\left(k_{\|} v \mu-\omega+n \Omega\right)^{2}}{k_{\|}^{2}\left(\Delta v_{\|}\right)^{2}}\right]
$$

where $n=0$ and \pm 1 (see YL08; YLP08).

Using Equations (C4) for (C1) combined with (C7), we obtain

$$
D_{p p}(\mu)^{\mathrm{G}}=\frac{v \sqrt{\pi} \Omega^{2}\left(1-\mu^{2}\right) m^{2} V_{\mathrm{A}}^{2} M_{\mathrm{A}}^{2}}{4 L R^{2}} \int_{1}^{k_{c} L} x^{-5 / 2} d x \int_{0}^{1} \frac{d \eta}{\eta \Delta \mu}\left[J_{0}^{2}(w)+J_{2}^{2}(w)\right] \exp \left[-\frac{\left(\mu-\frac{V_{A}}{\eta v} \pm \frac{1}{\eta x R}\right)^{2}}{\Delta \mu^{2}}\right],
$$

for the gyro-resonant acceleration $n= \pm 1$, and $d^{3} k=2 \pi k^{2} d k d \eta$ has been used for fast modes. In the above equation, $L$ is the injection scale of turbulence, $w=k_{\perp} v_{\perp} / \Omega, x=k / k_{\min }=k L, R=v k_{\min } / \Omega, M_{\mathrm{A}}^{2}=\delta V^{2} / V_{\mathrm{A}}^{2}, \eta=\cos \theta, k_{c}$ is the cut-off of turbulence cascade due to damping, and $J_{n}$ is second order Bessel function.

For the transit time acceleration (TTD), $n=0$, we obtain

$$
D_{p p}(\mu)^{\mathrm{TTD}}=\frac{v \sqrt{\pi} \Omega^{2}\left(1-\mu^{2}\right) m^{2} V_{\mathrm{A}}^{2} M_{\mathrm{A}}^{2}}{2 L R^{2}} \int_{1}^{k_{c} L} x^{-5 / 2} d x \int_{0}^{1} \frac{d \eta}{\eta \Delta \mu} J_{1}^{2}(w) \exp \left[-\frac{\left(\mu-\frac{V_{\mathrm{A}}}{\eta v}\right)^{2}}{\Delta \mu^{2}}\right],
$$

where $L$ is the injection scale of turbulence, $w=k_{\perp} v_{\perp} / \Omega, x=k / k_{\min }=k L, R=v k_{\min } / \Omega, M_{\mathrm{A}}^{2}=\delta V^{2} / V_{\mathrm{A}}^{2}$.

In the QLT, $R_{n}$ is replaced by $\delta$ function, Equations (C8) and (C9) become

$$
D_{p p}(\mu)^{\mathrm{G}}=\frac{v \Omega^{2}\left(1-\mu^{2}\right) m^{2} V_{\mathrm{A}}^{2} M_{\mathrm{A}}^{2}}{4 L R^{2}} \int_{1}^{k_{c} L} x^{-5 / 2} d x \int_{0}^{1} \frac{d \eta}{\eta}\left[J_{0}^{2}(w)+J_{2}^{2}(w)\right] \delta\left(\mu-\frac{V_{A}}{\eta v} \pm \frac{1}{\eta x R}\right),
$$

and

$$
D_{p p}^{\mathrm{TTD}}=\frac{v \Omega^{2}\left(1-\mu^{2}\right) m^{2} V_{\mathrm{A}}^{2} M_{\mathrm{A}}^{2}}{2 L R^{2}} \int_{1}^{k_{c} L} x^{-5 / 2} d x \int_{0}^{1} \frac{d \eta}{\eta}\left[J_{1}^{2}(w)\right] \delta\left(\mu-\frac{V_{A}}{\eta v}\right) .
$$

\section{REFERENCES}

Armstrong, J. W., Rickett, B. J., \& Spangler, S. R. 1995, ApJ, 443, 209

Beresnyak, A. 2011, Phys. Rev. Lett., 106, 075001 
Beresnyak, A., \& Lazarian, A. 2010, ApJ, 722, 110

Chandran, B. D. G., Li, B., Rogers, B. N., Quataert, E., \& Germaschewski, K. 2010, ApJ, 720, 503

Chepurnov, A., \& Lazarian, A. 2010, ApJ, 710, 853

Cho, J., \& Lazarian, A. 2002, Phys. Rev. Lett., 88, 245001

Cho, J., Lazarian, A., \& Vishniac, E. T. 2002, ApJ, 564, 291

Cho, J., \& Lazarian, A. 2003, MNRAS, 345, 325

Chokshi, A., Tielens, A. G. G. M., Hollenbach, D. 1993, ApJ, 407, 806

Dolginov, A. Z., \& Mitrofanov, I. G. 1976, Ap\&SS, 43, 291

Draine, B. T. 1985, Protostars and Planets II, 621

Draine, B. T. 2011, Physics of the Interstellar and Intergalactic Medium (Princeton, NJ: Princeton Univ. Press)

Draine, B. T., \& Lazarian, A. 1998, ApJ, 508, 157

Draine, B. T., \& Salpeter, E. E. 1979, ApJ, 231, 438

Draine, B. T., \& Sutin, B. 1987, ApJ, 320, 803

Draine, B. T., \& Weingartner, J. 1996, ApJ, 470, 551

Dullemond, C. P., \& Dominik, C. 2005, A\&A, 434, 971

Dung, R., \& Schlickeiser, R. 1990a, A\&A, 237, 504

Dung, R., \& Schlickeiser, R. 1990b, A\&A, 240, 537

Fisk, L. A. 1976, J. Geophys. Res., 81, 4633

Goldreich, P., \& Sridhar, S. 1995, ApJ, 438, 763

Hirashita, H., \& Yan, H. 2009, MNRAS, 394, 1061

Hirashita, H., Nozawa, T., Yan, H., \& Kozasa, T. 2010, MNRAS, 404, 1437

Hoang, T., Draine, B. T., \& Lazarian, A. 2010, ApJ, 715, 1462

Hoang, T., \& Lazarian, A. 2011, ApJ, submitted

Hoang, T., Lazarian, A., \& Draine, B. T. 2011, ApJ, 741, 87

Ivlev, A. V., Lazarian, A., Tsytovich, V. N., de Angelis, U.,

Hoang, T., Morfill, G. E. 2010, ApJ, 723, 612
Jokipii, J. R. 1966, ApJ, 146, 480

Jordan, M. E., \& Weingartner, J. C. 2009, MNRAS, 400, 536

Kowal, G., \& Lazarian, A. 2010, ApJ, 720, 742

Lazarian, A. 2007, J. Quant. Spectrosc. Rad. Trans., 106, 225

Lazarian, A. 1995, ApJ, 453, 229

Lazarian, A., \& Hoang, T. 2007a, MNRAS, 378, 910

Lazarian, A., \& Hoang, T. 2007b, ApJ, 669, L77

Lazarian, A. 2009, Space Sci. Rev., 143, 357

Lazarian, A., \& Yan, H. 2002, ApJ, 566, L105

Lithwick, Y., \& Goldreich, P. 2001, ApJ, 562, 279

Purcell, E. M. 1969, Physica, 41, 100

Roberge, W. G., Degraff, T. A., \& Flaherty, J. E. 1993, ApJ, 418, 287

Roberge, W. G., \& Lazarian, A. 1999, MNRAS, 305, 615

Schlickeiser, R., \& Miller, J. A. 1998, ApJ, 492, 352

Schlickeiser, R. 2002, Cosmic Ray Astrophysics (Berlin: Spinger)

Shalchi, A. 2005, Phys. Plasmas, 12, 052905

Völk, H. J. 1975, Rev. Geophys. Space Phys. 13, 547

Yan, H., \& Lazarian, A. 2003, ApJ, 592, L33

Yan, H., Lazarian, A., \& Draine, B. T. 2004, ApJ, 616, 895 (YLD04)

Yan, H., \& Lazarian, A. 2008, ApJ, 673, 942 (YL08)

Yan, H., \& Lazarian, A., \& Petrosian, V. 2008, ApJ, 684, 1461 (YLP08)

Yan, H. 2009, MNRAS, 397, 1093

Weingartner, J. C.,\& Draine, B. T. 2001, ApJS, 134, 263 